\title{
Personal Exposimeter for Radiation Assessment in Real Environments in the $60-\mathrm{GHz}$ Band
}

\author{
Reza Aminzadeh ${ }^{1}$, Arno Thielens ${ }^{1}$, Haolin $\mathrm{Li}^{1}$, Carole Leduc ${ }^{2}$, \\ Maxim Zhadobov ${ }^{2}$, Guy Torfs ${ }^{1}$, Johan Bauwelinck ${ }^{1}$, \\ Luc Martens ${ }^{1}$ and Wout Joseph ${ }^{1}$ \\ ${ }^{1}$ Department of Information Technology, Ghent University/iMinds, \\ Technologiepark-Zwijnaarde 15, B-9052 Ghent, Belgium \\ ${ }^{2}$ Institute of Electronics and Telecommunications of Rennes (IETR), \\ UMR CNRS 6164, University of Rennes 1, 35042 Rennes, France
}

\begin{abstract}
For the first time, a personal exposimeter (PE) for $60 \mathrm{GHz}$ radiation measurements is presented. The PE is designed based on numerical simulations and both on-body and on-phantom calibration measurements to determine the antenna aperture and measurement uncertainty of the PE. The measurement uncertainty of the PE is quantified in terms of $50 \%$ and $95 \%$ prediction intervals of its response. A PE consisting of three nodes (antennas) with VHH (vertical-horizontal-horizontal) polarization results in a $95 \%$ prediction interval of $6.6 \mathrm{~dB}$. A $50 \%$ prediction interval of $1.3 \mathrm{~dB}$ (factor of 1.3) is obtained for measured power densities which is $3.1 \mathrm{~dB}$ lower than a single antenna experiment. The uncertainty is $19.7 \mathrm{~dB}$ smaller than that of existing commercial exposimeters at lower frequencies $(\leq 6 \mathrm{GHz})$.
\end{abstract}

\section{Introduction}

Recent advances in $60 \mathrm{GHz}$ communication systems have increased the interest of the research community in mm-wave interactions with the human body [1,2]. This growing interest is associated with the concerns related to potential health effects. Safe usage of mm-wave systems requires them to be compliant with international guidelines, such as those issued by the International Commission on Non-Ionizing Radiation Protection (ICNIRP) [3]. The absorption of mm-waves is superficial [1], therefore, incident power density (IPD) is used as a dosimetric quantity. For far-field exposures, the IPD limits are $1 \mathrm{~mW} / \mathrm{cm}^{2}$ (general public) and $5 \mathrm{~mW} / \mathrm{cm}^{2}$ (occupational exposure) averaged over $20 \mathrm{~cm}^{2}$ of the exposed area [3]. Personal exposimeters (PEMs) have been used to measure the IPD in the 0.1-6 GHz range [4-6], for which a protocol has been developed [7]. PEMs' measurements are associated with a relatively large measurement uncertainty $[8,9]$ as they measure the total electric fields near the body instead of the incident electric fields or the IPD for which exposure limits are issued [3]. Recently, the interaction of a mobile terminal (phone call scenario) has been studied numerically at $60 \mathrm{GHz}$ [10]. Moreover, previous dosimetric studies at these frequencies have been mainly focused on cells and animals exposure assessment $[11,12]$. Recently, textile antennas [13] have been developed that allows for the development of wearable $60 \mathrm{GHz}$ applications.

In this paper, for the first time, a prototype of wearable on-body personal exposimeter (PE) for 
the mm-wave band is presented. The PE consists of three nodes (receiving antennas) to measure the incident power density in realistic indoor environments, and is calibrated in anechoic conditions using a real human subject. The measurements and simulations are used to determine the measurement uncertainty of the proposed prototype.

\section{Materials and methods}

Firstly, the PE is studied based on numerical simulations at $61 \mathrm{GHz}$. Secondly, the on-body calibration measurements on a real human subject are performed (at $61 \mathrm{GHz}$ ) to estimate its measurement uncertainty. The frequency range of the PE is 58$63 \mathrm{GHz}$.

\subsection{Antenna}

The antenna used in this study (Figure 1) is a microstrip-fed four-patch single-layer antenna array, operating in the $60-\mathrm{GHz}$ band [14]. The antenna is printed on a thin RT Duroid 5880 substrate $\left(\mathrm{h}=127 \mu \mathrm{m}, \epsilon_{r}=2.2, \tan \delta=0.003\right)$. The antenna has a total radiation efficiency of $60 \%$, gain of $11.8 \mathrm{dBi}$ and directivity of $13.9 \mathrm{dBi}$ (at $60 \mathrm{GHz}$ ) in free space [14].

\subsection{Numerical modeling}

Figure 1 demonstrates the considered numerical model. The goal of the simulations is to determine the distribution of the on-body received power on the antenna. The finite-difference time-domain (FDTD) method is used for simulations. Since in the $60-\mathrm{GHz}$ band the penetration depth into the skin is limited to $0.5 \mathrm{~mm} \mathrm{[1]}$, a homogeneous skin model is proposed $\left(200 \times 200 \times 10 \mathrm{~mm}^{3}\right)$ for simulations to emulate the human body. The dielectric properties used for this model are taken from [15] (dry skin at $61 \mathrm{GHz}: \epsilon_{r}=7.86, \sigma=36.5 \mathrm{~S} / \mathrm{m}$ ). A non-uniform mesh with maximum grid step of $100 \mu \mathrm{m}$ is used to resolve the skin model. The antenna is placed at $5.6 \mathrm{~mm}$ from the surface of

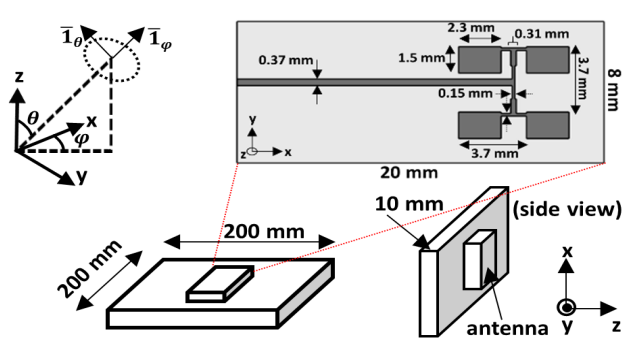

Figure 1: The proposed model for numerical simulations. Top: schematic representation of the antenna [14] (at $5.6 \mathrm{~mm}$ from the skin) used for simulations and measurements. Bottom: homogeneous skin model below the antenna.

the skin model, due to the geometry of the connector used in measurements. The connector and cable are not modeled. The absorbing boundary conditions are set to uniaxial perfectly matched layer (U-PML) with a thickness of 11 layers in the $+\mathrm{Z}$ direction and 8 layers in the other directions. The simulation time is set to 50 periods at $61 \mathrm{GHz}$, to reach a steady state condition. The entire geometry of the skin model and the antenna is meshed by $197 \times 10^{6}$ voxels.

In order to determine the response of the antenna, first, a free space simulation is performed for the antenna only. In the second step, simulations are performed for the antenna near the skin model $(5.6 \mathrm{~mm})$. The quantity studied here is the response $(R)$ of the antenna, which is the ratio of the median received power on-body $\left(P_{r}^{b o d y}\right)$ to the median received power in free space $\left(P_{r}^{\text {free }}\right)$ :

$$
R=\frac{P_{r}^{\text {body }}}{P_{r}^{\text {free }}}
$$

The received power on an antenna can be determined from its aperture [16]:

$$
P_{r}(\varphi, \theta)=A A(\varphi, \theta) \times S_{i n c}
$$

where $A A$ and $S_{i n c}$ are the on-body antenna aperture and incident power density, respectively. In order to calculate the on-body aperture of the antenna $A A(\varphi, \theta)$, the directive gain of the antenna 
$D(\varphi, \theta)$ is extracted for azimuth angle $(\varphi)$ and polar angle $(\theta)$ from numerical simulations as:

$$
A A(\varphi, \theta)=\eta_{\text {rad }}\left(1-\left|S_{11}\right|^{2}\right) D(\varphi, \theta) \frac{\lambda^{2}}{4 \pi}
$$

where $\eta_{\text {rad }}$ is the radiation efficiency, $\left|S_{11}\right|^{2}$ is the antenna's power reflection coefficient, and $\lambda$ is the wavelength. $A A(\varphi, \theta)$ can be determined for two orthogonal polarizations on the incident electric fields: $\theta$ and $\varphi$, which are the polarizations parallel to the unity vectors $\overline{1}_{\theta}$ and $\overline{1}_{\phi}$. For multiple plane waves incident on the antenna, the received power on the antenna is not necessarily equal to the sum of the incident powers induced by each single plane wave, since the incident plane waves can interfere with each other. Therefore, the received power $\left(P_{r}\right)$ is calculated as a function of the incident electric fields (the sum of the induced voltages on the antenna) $[6,17]$.

A realistic far-field exposure scenario in the 60$\mathrm{GHz}$ band for an indoor environment (conference room of IEEE 802.11 standard [18]) is considered to determine the response of the simulated on-body antenna near the skin model. A Gaussian distribution with a cross polarization ratio of $20 \mathrm{~dB}$ is assumed for the indoor exposure scenario [18]. Also the phase $(\alpha)$ of plane waves is assumed to have a uniform distribution in the range of $0<\psi<2 \pi$. For $\theta$ and $\varphi$, samples are drawn from their distributions [18].

\subsection{Calibration measurement setup}

Figure 2 shows the measurement setup at $61 \mathrm{GHz}$. The on-body calibration consists of two types of measurements: free space and on-body (on a male subject's forearm and on a skin-equivalent phantom for comparison). The measurements are performed on an optical table in a laboratory environment. Absorbers are placed on the surface of the table and around the antennas to emulate anechoic conditions and to minimize the influence of the table on the measurements. A standard gain (19 dBi) horn antenna (QMS-00475, Steatite Q-Par
Antennas, UK) is used as transmitter (TX) and is mounted on a rotation stage (CR1/M, Thorlabs). The microstrip-fed antenna used for simulations is used as a receiver (RX) and is placed in the far field of the TX $(49 \mathrm{~cm})$. An Agilent PNA-X N5247A network analyzer is used to deliver a constant power $(6 \mathrm{dBm})$ to the TX and to measure the on-body received power on the RX. The VNA has a dynamic range of $118 \mathrm{~dB}$ in the range of 58-63 GHz. The RX is fed with a V-connector that has a loss of about $0.8 \mathrm{~dB}$ [19]. Due to the asymmetric shape of RX and to account for any realistic polarization of TX, two orthogonal polarizations of the antennas are studied, horizontal $(\mathrm{H})$ parallel to the surface of the table and vertical $(\mathrm{V})$ perpendicular to this surface.

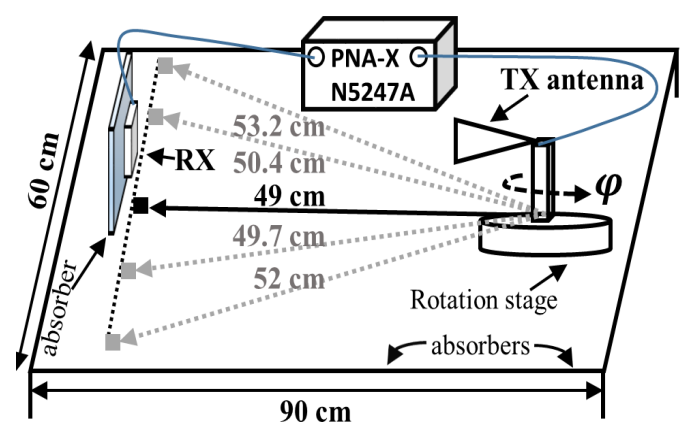

Figure 2: Measurement setup at $61 \mathrm{GHz}$. The surface of the table and around the antennas is covered with absorbers. Solid squares show five locations of RX.

In the first step, the free-space incident power density $S_{i n c}^{f r e e}$ is calculated using the Friis formula [16].

$$
S_{\text {inc }}^{\text {free }}=\frac{P_{t}}{4 \pi R^{2}} G_{t}
$$

Where $P_{t}$ is the output power of TX, $R$ the distance between TX and RX and $G_{t}$ is the gain of the TX. The free-space incident power density is then averaged over $20 \mathrm{~cm}^{2}$ of the studied area (on the 5 positions) [3].

In the second step, the RX is placed horizontally on a semi-solid phantom that mimicks the 
dielectric properties of human skin in the measured frequency bands. This phantom has been proposed in [20] for the frequency range of 26.5 to $40 \mathrm{GHz}$. Recently, it has been shown that this phantom can emulate the dielectric properties of human skin up to $100 \mathrm{GHz}$ with maximum $15 \%$ deviation [21] compared to [22] with $10 \%$ deviation. In this paper, the phantom is fabricated by mixing 428.5 grams gelatin powder (240 bloom, Carl Roth $\mathrm{GmbH}$ ) with 1000 grams de-ionized water following the instructions proposed in [20]. The S-parameters of TX and RX (on the phantom) are measured and the received power on the RX is calculated from the measured power gain $\left(\left|S_{21}\right|^{2}\right)$ at five positions as a function of azimuth $(\varphi)$ as shown in Figure 2 (solid squares) for both $\mathrm{H}$ and $\mathrm{V}$ polarizations of the TX. Before every measurement on each position, the TX is rotated toward the RX $(\varphi)$ until the maximum received power on the $\mathrm{RX}$ is measured. This ensures that the main beam of the TX is directed toward the RX.

In the third step, the RX is placed on a male subject's forearm and the received power is measured on the RX at five locations similar to the second step (see Figure 2). Both polarizations of TX and RX are studied.

In the fourth step, three locations are selected for mounting the RX on the forearm (see Figure 3) with a distance of $1 \lambda$ and the received power is measured as a function of $(\varphi)$ resulting in a distribution for the measured received power on each antenna:

$$
R_{\text {meas }}(\varphi)=10 \times \log \left(\frac{\frac{1}{N} \sum_{i=1}^{N}\left(P_{r, i}^{b o d y}(\varphi)\right)}{P_{r}^{\text {free }}}\right)
$$

where $R_{\text {meas }}$ is the response, $P_{r, i}^{b o d y}(\varphi)$ is the onbody received power on each antenna $i$.

Considering two polarizations for the TX and $\mathrm{RX}, 12$ measurements are performed and the best combination of the three antennas on the forearm is determined based on the $50 \%$ prediction interval $\left(\mathrm{PI}_{50}\right)$ of the measurements. The on-body received

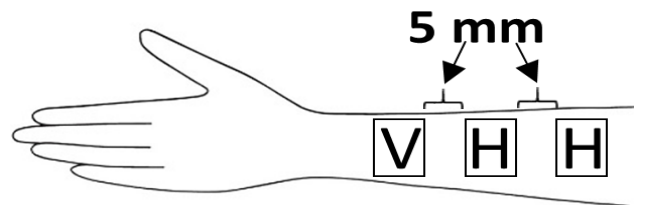

Figure 3: The optimized orientation of the antennas on the subject's forearm determined from the on-body calibration measurements.

power is averaged over the three antennas which reduced the $\mathrm{PI}_{50}$ of the response

\subsection{Using calibration data to process mea- surements in a real environment}

During the measurements in the real environment a power $\left(P_{r, i}^{\text {meas }}\right)$ is recorded on each antenna $i$. This received power can be used to determine the incident power density $\left(S_{\text {inc }}\right)$ :

$$
S_{i n c}=\frac{P_{r, i}^{\text {meas }}}{A A_{i}}
$$

where $A A_{i}\left(\mathrm{~m}^{2}\right)$ is the effective median onbody antenna aperture obtained from calibration measurements. During the calibration measurements, the received powers on different antennas $i, P_{r, i}^{b o d y, H / V}(\varphi)$, are recorded on the body for two orthogonal $(\mathrm{H}$ and $\mathrm{V})$ polarizations of the TX at a constant input power. The free-space power density $S_{i n c}^{f r e e, H / V}$ for the same input power is obtained from the first step of calibration measurements. Next, $A A_{i}(\varphi, \psi)$ values are determined for any realistic polarization (as the the sum of two orthogonal polarizations) [17]:

$$
\begin{aligned}
A A_{i}(\varphi, \psi) & =\frac{P_{r, i}^{\text {body }, H}(\varphi)}{S_{i n c}^{\text {free }, H}} \cos ^{2}(\psi) \\
& +\frac{P_{r, i}^{\text {body }, V}(\varphi)}{S_{i n c}^{f r e e, V}} \sin ^{2}(\psi)
\end{aligned}
$$

A Gaussian distribution that is applicable for indoor $60 \mathrm{GHz}$ communications is used [18]. 
During the calibration measurements the powers $P_{r, i}^{b o d y, H / V}(\varphi)$ are registered for five angles $(\varphi)$ as depicted in Figure 2: $-20^{\circ},-10^{\circ}, 0^{\circ}, 13^{\circ}$ and $23^{\circ}$. The antenna apertures $A A_{i}$ are then calculated using (7). These antenna apertures $A A_{i}$ are determined for a realistic polarization $\psi$. Next, $1000 \psi$ samples are generated for every measured power value and this is repeated during 1000 iterations. Calculating $A A_{i}$, for every pair of $(\varphi, \psi)$, results in a distribution of $A A_{i}$ for every antenna $i$.

\section{Results and discussion}

Figure 4 shows the simulated and measured (2048 points) power reflection coefficient $(55-65 \mathrm{GHz})$ of the antenna in free space and on-body as well as on the skin phantom and skin model. The measured reflection coefficient $\left(\left|S_{11}\right|\right)$ remained almost the same for RX distances smaller than $5.6 \mathrm{~mm}$ (depending on the location of the nodes). At $61 \mathrm{GHz}$, the simulated antenna has a total radiation efficiency of $76 \%$, a gain of $13.6 \mathrm{dBi}$ and a directivity of $14.1 \mathrm{dBi}$ in free space. The difference between measurement and simulation can be explained by the absence of connector and cable in the proposed numerical model as well as possible fabrication error of the antenna.

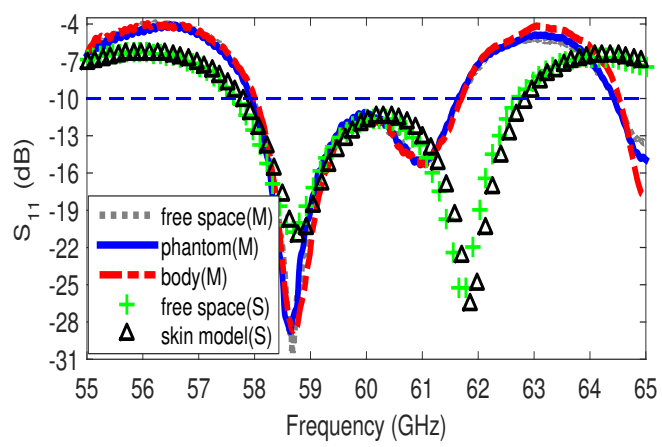

Figure 4: Simulated (S) and measured (M) power reflection coefficients for the antenna in free space and on body, skin phantom and the numerical model.

The measured incident free space power density
Table 1: The on-body antenna aperture obtained from simulations and measurements.

\begin{tabular}{ccc}
\hline Scenario & AA $\left(\mathrm{mm}^{2}\right)$ & difference* $\left.^{*} \%\right)$ \\
\hline Simulation (H) $^{a}$ & 0.077 & 17.7 \\
${\text { body }(\mathrm{H})^{b}}^{b}$ & 0.092 & 0 \\
phantom (H) $^{a}$ & 0.1 & 8.3 \\
${\text { body }(\mathrm{V})^{a}}^{a}$ & $23 \times 10^{-3}$ & 199 \\
\hline
\end{tabular}

${ }^{*}[|a-b| /(0.5 \times(a+b))] \times 100$

(on the location of RX and at the same height as $\mathrm{TX})$ is $2.4 \mu \mathrm{W} / \mathrm{cm}^{2}$. Table 1 shows the median onbody antenna aperture obtained from calibration measurements for both $\mathrm{H}$ and $\mathrm{V}$ polarizations of the TX and RX. The measured antenna aperture for the horizontally $(\mathrm{H})$ oriented RX on-body and on the skin equivalent phantom are in good agreement (8.3\% difference body vs phantom and $17.7 \%$ acceptable agreement for body vs simulation). Due to the exclusion of the connector in simulations (lower power loss), simulated AA is higher than measured AA. Also, the results suggest that the absorbed energy for the body is slightly higher than the phantom which leads to a lower received power on the antenna on body and thus a lower AA value for the body with respect to the phantom.

The difference between the determined antenna aperture for two $\mathrm{H}$ and $\mathrm{V}$ polarizations of the $\mathrm{RX}$ in Table 1 is due to the asymmetric structure of the RX. This is due to the different attenuation between two polarizations at $\mathrm{mm}$-wave bands and strong dependence of reflection and transmission coefficients on the angle of incidence [1,2].

The simulated median received power on the antenna is $1.2 \mathrm{nW}$ for the antenna at $5.6 \mathrm{~mm}$ from the skin model and $1.6 \mathrm{nW}$ in free space. Figure 5 shows the on-body measured received power for $\mathrm{V}$ and $\mathrm{H}$ polarizations of the RX and TX on the phantom and forearm.

The response is determined using (1) and is equal to $0.8(-0.96 \mathrm{~dB}), 0.75(-1.25 \mathrm{~dB})$ and $0.72(-$ 


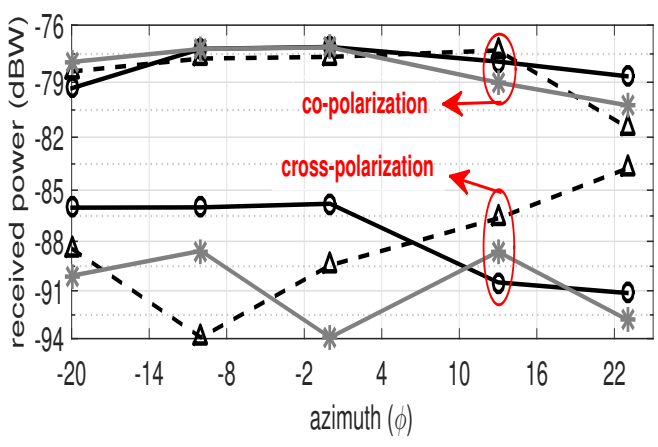

Figure 5: The measured on-body received power on RX for different polarizations during calibration measurements (on the skin-equivalent phantom and human forearm). black lines: on-body $(\mathrm{H})$; gray lines: on-phantom $(\mathrm{H})$, dashed lines: on$\operatorname{body}(\mathrm{V})$

$1.4 \mathrm{~dB}$ ) for one-antenna calibration measurements on body, measurements on the phantom and for simulations on the phantom, respectively. This indicates underestimation of the incident electric fields with respect to free space values. This conclusion is also obtained for exposure assessment at frequencies $\leq 6 \mathrm{GHz}[6,8]$.

Figure 6 compares the lowest simulated and measured $\mathrm{PI}_{50}$ and $\mathrm{PI}_{95}$ (which are measures for uncertainty of PE) of the response $\left(\mathrm{R}_{\text {meas }}(\varphi)\right)$ of the PE consisting of 1, 2 and 3 antennas, according to the best combination of three antennas on the subject's forearm as shown in Figure 3.

For simulations, the received power $P_{r}$ (see Section 'Numerical Modeling') is calculated from the on-body antenna aperture for 1, 2 and 3 antennas on the numerical skin model (Figures 1 and 3 ). The antennas are placed on the skin as shown in figure 3. The value of simulated $\mathrm{PI}_{95}$ is reduced from $21.4 \mathrm{~dB}$ to $17.6 \mathrm{~dB}$ and $12.2 \mathrm{~dB}$ for three, two and one antenna, respectively. Also the $\mathrm{PI}_{50}$ value is reduced from $7 \mathrm{~dB}$ (1 antenna) to 5 (2 antennas) and $3.2 \mathrm{~dB}$ (3 antennas), which is an improvement of $3.8 \mathrm{~dB}$.

For the measurements, The value of $\mathrm{PI}_{50}$ is reduced from $4.4 \mathrm{~dB}$ to $3.6 \mathrm{~dB}$ and $1.3 \mathrm{~dB}$ for three, two and one antenna, respectively. Thus an improvement of $3.1 \mathrm{~dB}$ is obtained. Also a $\mathrm{PI}_{50}$ of $3.1 \mathrm{~dB}$ on $S_{i n c}$ has been reported for a PE at lower frequencies $(\leq 6 \mathrm{GHz})$ [23] which is $1.8 \mathrm{~dB}$ higher than the $\mathrm{PI}_{50}$ of our proposed exposimeter at $61 \mathrm{GHz}$. Our proposed exposimeter has an improved $\mathrm{PI}_{50}$ of $16.6 \mathrm{~dB}$ (1 antenna) and $19.7 \mathrm{~dB}$ (3 antennas) compared to the $\mathrm{PI}_{50}$ of commercial PEMs (up to $21 \mathrm{~dB}$ ) at lower frequencies [8]. Also the $\mathrm{PI}_{95}$ value is reduced from $15.4 \mathrm{~dB}$ (1 antenna) to $6.6 \mathrm{~dB}$ (2 and 3 antennas), which is an $8.8 \mathrm{~dB}$ improvement. The $\mathrm{PI}_{95}$ is $11.9 \mathrm{~dB}$ lower than that of a commercial exposimeter in an indoor scenario (18.5 dB at $900 \mathrm{MHz})$ [9].

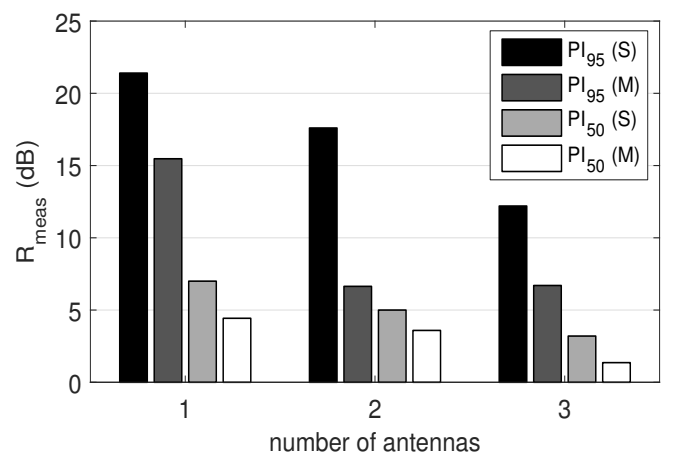

Figure 6: The response with smallest $50 \%$ and 95\% prediction intervals obtained from simulations (S) and on-body calibration measurements (M).

\section{4 conclusion}

A mm-wave personal exposimeter is proposed using a limited number of wearable antennas. All results, whether using numerical simulations or calibration measurements, show that using a combination of multiple antennas, the proposed exposimeter has good accuracy. An improvement of 3-9 dB is obtained (from simulations and measurements) for three antennas compared to a single antenna experiment and $19.7 \mathrm{~dB}$ compared to the commercial exposimeters at lower frequencies $(\leq 6 \mathrm{GHz})$. The 
proposed prototype is the first $60 \mathrm{GHz} \mathrm{PE}$ and to the authors' best knowledge no data are available in the literature. Future work will consist of developing the PE for an improved wearability and considering diffuse fields exposure in a $60 \mathrm{GHz}$ reverberation chamber.

\section{Funding}

Research Foundation - Flanders (FWO-V); grant agreement No G027714N.

\section{References}

[1] M. Zhadobov, N. Chahat, R. Sauleau, C. LeQuement, and Y. Le Drean. Millimeter-wave interactions with the human body: state of knowledge and recent advances. Int J Microw Wirel T., 3(02):237-247, 42011.

[2] Ting W., T.S. Rappaport, and C.M. Collins. Safe for generations to come: Considerations of safety for millimeter waves in wireless communications. IEEE Microw. Mag., 16(2):65-84, March 2015.

[3] International Commission on Non-ionizing Radiation Protection (ICNIRP). Guidelines for limiting exposure to time-varying electric, magnetic, and electromagnetic fields (up to $300 \mathrm{GHz}$ ). Health Phys., 74(4):494-522, 1998.

[4] P. Frei, E. Mohler, G. Neubauer, G. Theis, A. Bürgi, J. Fröhlich, C. Braun-Fahrländer, J. Bolte, M. Egger, and M. Röösli. Temporal and spatial variability of personal exposure to radio frequency electromagnetic fields. Environ. Res., 109(6):779-785, 2009.

[5] A. Thielens, S. Agneessens, L. Verloock, E. Tanghe, H. Rogier, L. Martens, and W. Joseph. On-body calibration and processing for a combination of two radio-frequency personal exposimeters. Radiat Prot Dosimetry, 163(1):58-69, 2015.

[6] R. Aminzadeh, A. Thielens, A. Bamba, L. Kone, D.P. Gaillot, M. Lienard, L. Martens, and W. Joseph. On-body calibration and measurements using personal radiofrequency exposimeters in indoor diffuse and specular environments. Bioelectromagnetics, 37(5):298-309, 2016.

[7] M. Röösli, P. Frei, J. Bolte, G. Neubauer, E. Cardis, M. Feychting, P. Gajsek, S. Heinrich, W. Joseph, S. Mann, L. Martens, E. Mohler, R. C. Parslow, A. H. Poulsen, K. Radon, J. Schuz, G. Thuroczy, J. F. Viel, and M. Vrijheid. Conduct of a personal radiofrequency electromagnetic field measurement study: proposed study protocol. Environ Health, 9:23, 2010.

[8] J.F. Bolte, G. van der Zande, and J. Kamer. Calibration and uncertainties in personal exposure measurements of radiofrequency electromagnetic fields. Bioelectromagnetics, 32(8):652-663, 2011.

[9] S. Iskra, R. McKenzie, and I. Cosic. Monte carlo simulations of the electric field close to the body in realistic environments for application in personal radiofrequency dosimetry. Radiat. Prot. Dosimetr., 147(4):517-527, 2011.

[10] A.R. Guraliuc, M. Zhadobov, R. Sauleau, L. Marnat, and L. Dussopt. Millimeter-wave electromagnetic field exposure from mobile terminals. In Networks and Communications (EuCNC), 2015 European Conference on, pages 82-85, June 2015.

[11] M. Zhadobov, R. Sauleau, R. Augustine, C. Le-Quément, Y. Le Dréan, and D. Thouroude. Near-field dosimetry for in vitro exposure of human cells at $60 \mathrm{GHz}$. Bioelectromagnetics, 33(1):55-64, 2012. 
[12] J. Zhao and H. Lu. Reduction of exposure inhomogeneity for millimeter-wave experiments on cells in vitro. IEEE Trans. Microw. Theory Techn., 63(2):533-545, Feb 2015.

[13] N. Chahat, M. Zhadobov, S.A. Muhammad, L. Le Coq, and R. Sauleau. 60-GHz textile antenna array for body-centric communications. IEEE Trans. Antennas Propag., 61(4):1816-1824, April 2013.

[14] N. Chahat, M. Zhadobov, L. Le Coq, S.I. Alekseev, and R. Sauleau. Characterization of the interactions between a $60-\mathrm{GHz}$ antenna and the human body in an off-body scenario. IEEE Trans. Antennas Propag., 60(12):5958-5965, Dec 2012.

[15] S. Gabriel, R.W. Lau, and C. Gabriel. The dielectric properties of biological tissues: III. parametric models for the dielectric spectrum of tissues. Phys. Med. Biol., 41, 1996.

[16] C. A. Balanis. Antenna Theory: Analysis and Design. Wiley-Interscience, 3 edition, 2005.

[17] A. Thielens, S. Agneessens, H. De Clercq, J. Lecoutere, L. Verloock, E. Tanghe, S. Aerts, R. Puers, H. Rogier, L. Martens, and W. Joseph. On-body calibration and measurements using a personal, distributed exposimeter for wireless fidelity. Health Phys., 108(4):407-418, 2015.

[18] A. Maltsev, V. Erceg, E. Perahia, Ch. Hansen, R. Maslennikov, A. Lomayev, A. Sevastyanov, A. Khoryaev, G. Morozov, M. Jacob, S. Priebe, Th. Kürner, Sh. Kato, H. Sawada, K. Sato, and H. Harada. Channel models for $60 \mathrm{GHz}$ WLAN Systems. Technical Report IEEE 802.11-09/0334r8, May 2010.

[19] N. Chahat, M. Zhadobov, L. Le Coq, and R. Sauleau. Wearable endfire textile antenna for on-body communications at 60
GHz. IEEE Antennas and Wireless Propagation Letters, 11:799-802, 2012.

[20] R. Aminzadeh, M. Saviz, and A.A. Shishegar. Theoretical and experimental broadband tissue-equivalent phantoms at microwave and millimetre-wave frequencies. Electronics Letters, 50(8):618-620, April 2014.

[21] J. Lacik, V. Hebelka, J. Velim, Z. Raida, and J. Puskely. Wideband skin-equivalent phantom for $\mathrm{V}$ and $\mathrm{W}$ band. IEEE Antennas Wireless Propag. Lett., (99), 2015.

[22] N. Chahat, M. Zhadobov, and R. Sauleau. Broadband tissue-equivalent phantom for ban applications at millimeter waves. IEEE Trans. Microw. Theory Techn., 60(7):22592266, July 2012.

[23] A. Thielens, P. Vanveerdeghem, S. Agneessens, P. Van Torre, G. Vermeeren, H. Rogier, L. Martens, and W. Joseph. Wholebody averaged specific absorption rate estimation using a personal, distributed exposimeter. IEEE Antennas Wireless Propag. Lett., 14:1534-1537, 2015. 